\title{
Composition and variation of the skin microbiota in sympatric species of European newts (Salamandridae)
}

\author{
Miguel Vences ${ }^{1}$, Anja B. Dohrmann ${ }^{2}$, Sven Künzel ${ }^{3,4}$, Susann Granzow ${ }^{1,2}$, \\ John F. Baines ${ }^{3,4}$, Christoph C. Tebbe ${ }^{2}$
}

1 - Zoological Institute, Technische Universität Braunschweig, Mendelssohnstr. 4, 38106

Braunschweig, Germany

2 - Thünen Institute of Biodiversity, Bundesallee 50, 38116 Braunschweig, Germany

3 - Max-Planck-Institute for Evolutionary Biology, 24306 Plön, Germany

4 - Institute for Experimental Medicine, Christian-Albrechts-University of Kiel, Kiel, Germany

\section{Supplementary Material}



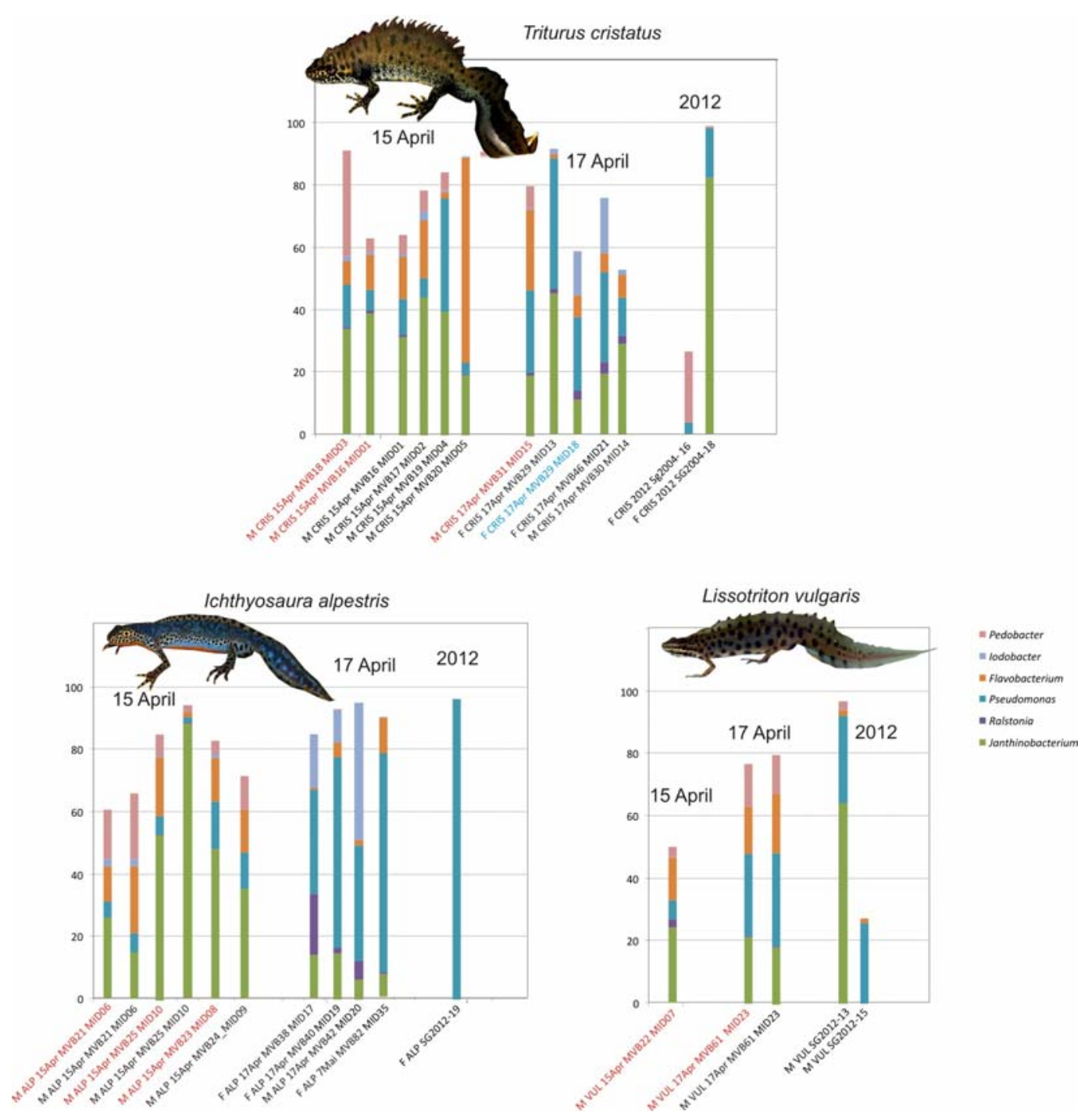

Figure S1. Extended version of Fig. 1, showing also repeated samples amplified by hemi-nested PCR, and the sex of each host specimen (M, male; F, female). All hemi-nested PCR samples are marked in red font, a repeat with normal (hotstart) PCR is marked in blue font. The figure shows the proportion of the six most common genera of bacteria among 16S rRNA sequences obtained from unique specimen samples of three species of newts (from 15 and 17 April 2013, and from 20 April 2012). Values from repeated samples in most cases agree very well with the original values of the same sample, suggesting repeatability of results. Although for Triturus and Ichthyosaura, the 15 April sampling contains mostly males and the 17 April sampling mostly females, several males at the second date (e.g., MVB 30, T. cristatus; MVB 42, I. alpestris) show a similar low proportion of Flavobacterium and and/or Janthinobacterium, and high proportion of Pseudomonas, as typical for the females from that date. Note that the additional samples collected in 2012 were amplified using the unlabelled primers 16S-27F and 16S-338R (GCTGCCTCCCGTAGGAGT) with slightly different PCR protocols, and library preparation and 454 sequencing outsourced to GATC (Konstanz, Germany). These data are here presented for completeness but not included in the statistical analyses. 


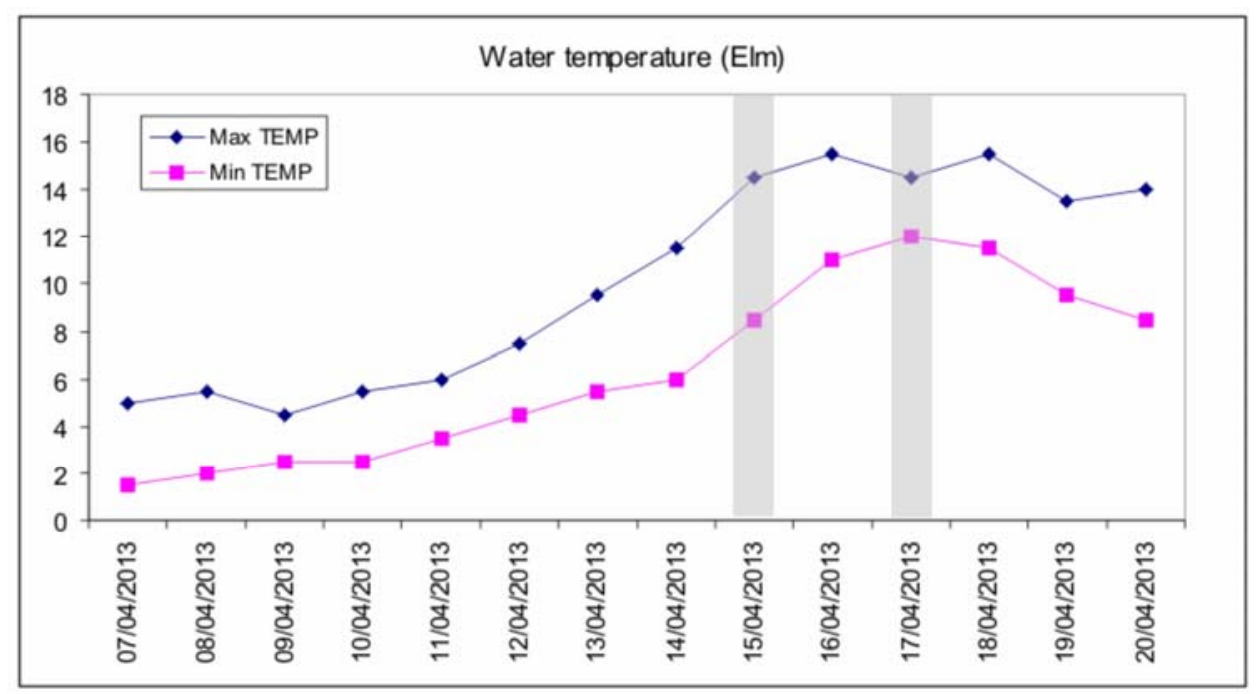

Figure S2. Minimum and maximum water temperatures recorded in the study pond by data loggers. Gray shading indicates the two sampling days. 